\title{
Environmental TEM Studies Reveal Catalyst/Support Registry on 2D Zeolites
}

Jennifer Carpena-Núñez, ${ }^{1,2,}{ }^{*}$ Dmitri Zakharov, ${ }^{2}$ Rahul Rao, ${ }^{1,3}$ Donghun Kim, ${ }^{4}$ J. Anibal Boscoboinik, ${ }^{2}$ Eric A. Stach, ${ }^{5}$ Michael Tsapatsis, ${ }^{6}$ Dario Stacchiola, ${ }^{2}$ and Benji Maruyama ${ }^{1}$

${ }^{1}$ Materials and Manufacturing Directorate, Air Force Research Laboratory, Wright-Patterson Air Force Base, Dayton, OH 45433.

${ }^{2}$ Center for Functional Nanomaterials, Brookhaven National Laboratory, Upton, NY 11973.

${ }^{3}$ UES, Inc., Dayton, OH 45431.

${ }^{4}$ Department of Chemical Engineering, University of Minnesota, Minneapolis, MN 55455.

${ }^{5}$ Department of Materials Science and Engineering, University of Pennsylvania, Philadelphia, PA 19104.

${ }^{6}$ Department of Chemical and Biomolecular Engineering, John Hopkins University, Baltimore, MA 21218.

\section{*Corresponding author: jennifer.carpena.ctr@us.af.mil}

One of the factors contributing to the slow progress in carbon nanotube (CNT) fields is the lack of understanding and control of catalyst evolution behavior. Lack of control over catalyst evolution may lead to unfavorable outcomes (i.e. catalyst coarsening and Ostwald ripening) which impede the growth of small-diameter CNTs. A strategy used to control catalyst diameter and evolution is to choose a catalyst support which hinders evolution. Examples of these are either bulk or porous oxides (i.e. silicon, aluminum, or magnesium).[1, 2] Porous aluminosilicates, namely zeolites, have been used to grow very small-diameter CNTs with control over chirality as well as diameter distribution by tuning the chemical state and diameter of the catalyst nanoparticles.[3-7]

Here, environmental transmission electron microscope (ETEM) was used to reveal how 2D zeolite supports bias the population density of catalyst nanoparticles. To do so, the catalyst/support system was monitored during the reducing and growth stages using conditions analog to chemical vapor deposition growth experiments $-\mathrm{H}_{2}$ for reduction, $\mathrm{C}_{2} \mathrm{H}_{2}$ for growth, a temperature of $850^{\circ}-900^{\circ} \mathrm{C}$, and a thin cobalt catalyst layer. The TEM micrographs obtained at $900^{\circ} \mathrm{C}$ (Figure 1a) were processed using fast Fourier transform (FFT) filtering and inversion procedures in Gatan's Digital Micrograph (DM) suite. [8] We extracted the components corresponding to the 2D zeolite films (Figure 1b) and the nanoparticles (Figure 1c) using either a spot filter (SF-) or band pass filter (BPF-), respectively. The inverted FFT (iFFT) images were then overlaid to yield composite images (Figure 1b) to highlight the catalyst/support registry.

ETEM micrographs showed that the 2D zeolites stabilized the catalyst nanoparticles, effectively hindering catalyst evolution (i.e. Ostwald ripening and coalescence) and promoting the subsistence of small-diameter nanoparticles. The formation of catalyst particles was seen to take place primarily during the reduction stage, with little evolution beyond reduction. The resulting nanoparticle population exhibited a very narrow size distributions. Image processing of micrographs collected at the onset of growth revealed that the nanoparticles were preferentially docked to a discrete number of pores -with docking to one, three, and four pores being the most prominent, suggesting that support registry biases nanoparticle evolution. The formation of nanoparticles with energetically-costly form factors (i.e. oval particles docked to two pores) on the zeolites further stresses the influence of the 2D zeolite support 
during evolution. These results reinforce the need for appropriate catalyst/support interactions to control CNT growth outcome.

\section{References:}

[1] PB Amama et al., ACS Nano 4 (2010), p. 895.

[2] PB Amama et al., Nano Lett 9 (2009), p. 44.

[3] E-H Kwak, KB Yoon and G-H Jeong, Current Applied Physics 14 (2014), p. 1633.

[4] T Moteki et al., The Journal of Physical Chemistry C 118 (2014), p. 23664.

[5] T Moteki et al., The Journal of Physical Chemistry C 115 (2011), p. 24231.

[6] H Ago et al., The Journal of Physical Chemistry B 103 (1999), p. 8116.

[7] B Xu et al., Sci Rep 7 (2017), p. 11149.

[8] Gatan Digital Micrograph Software, http://www.gatan.com/products/tem-analysis/gatan-microscopysuite-software
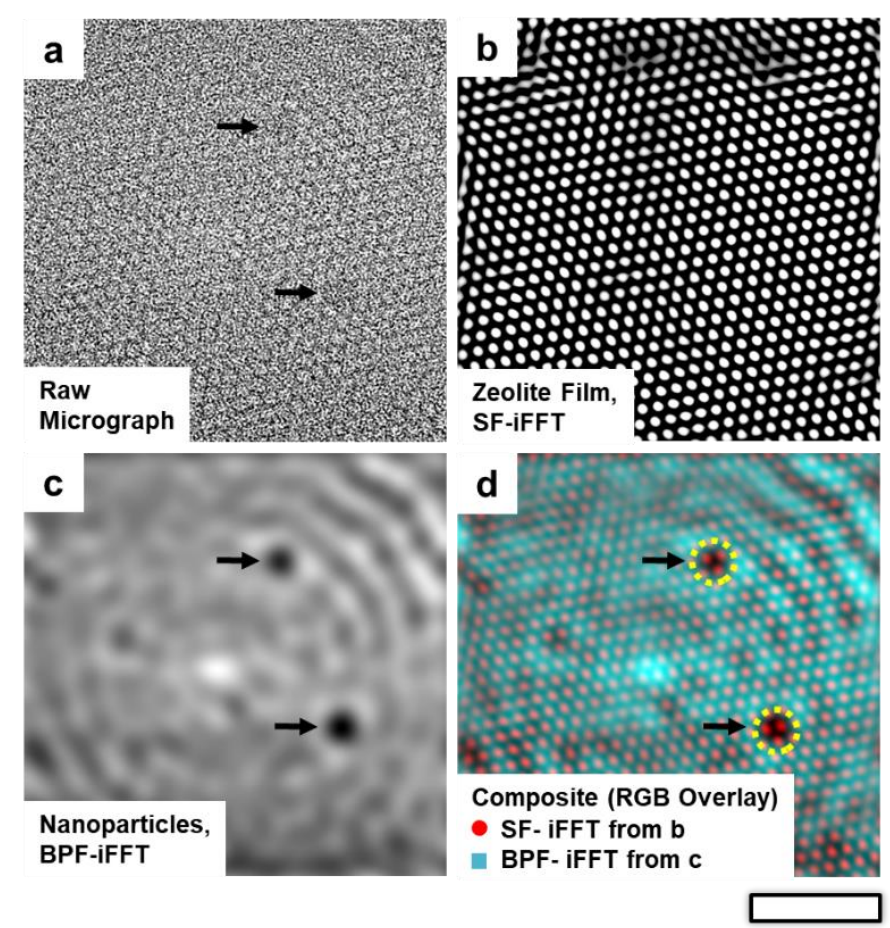

Figure 1. Zeolite registry biases nanoparticle diameters. The raw (a), filtered (d-c), and composite (d) micrographs show the docking of catalyst nanoparticles to three pores of the 2D zeolite. Panels b and c correspond to the spot filtered (SF-) and band pass filtered (BPF-) inverse FFTs (iFFTs); these highlight the zeolite pores (b) and catalyst nanoparticles (c). The composite RGB (d) obtained from overlaying (b) and (c) emphasizes the catalyst/support registry. The scale bar below panel d corresponds to 10nm. 